\title{
Damping Properties of Vibrations of Three-Layer VIscoelastic Plate
}

\author{
Safarov Ismail Ibrahimovich ${ }^{1}$, Teshayev Muhsin Khudoyberdiyevich ${ }^{2}$, \\ Boltayev Zafar Ixtiyorovich ${ }^{2}$, Akhmedov Maqsud Sharipovich ${ }^{2}$ \\ ${ }^{1}$ Department of "Mathematics", Tashkent Khimical-Technological Institute, Tashkent, Uzbekistan \\ ${ }^{2}$ Department of "Mathematics", Bukhara Engineering-Technological Institute, Bukhara, Uzbekistan
}

Email address:

Muhsin_5@mail.ru (T. M. Khudoyberdiyevich)

\section{To cite this article:}

Safarov Ismail Ibrahimovich, Teshayev Muhsin Khudoyberdiyevich, Boltayev Zafar Ixtiyorovich, Akhmedov Maqsud Sharipovich. Damping Properties of Vibrations of Three-Layer VIscoelastic Plate. International Journal of Theoretical and Applied Mathematics.

Vol. 3, No. 6, 2017, pp. 191-198. doi: 10.11648/j.ijtam.20170306.13

Received: September 28, 2017; Accepted: November 3, 2017; Published: November 30, 2017

\begin{abstract}
The work is devoted to the study of harmonic waves in a hereditarily elastic plate with two viscoelastic coatings, the properties of the material, which are described by the equations of state in integral form. The fractional exponential function of Rabotnov and Koltunov-Rzhanitsyn was chosen as the kernel of the integral operator. Two cases are considered: the case of a stress-strain state symmetric and antisymmetric in the normal coordinate (VAT). In the study of natural oscillations, the properties of those modes that are time-dependent by harmonic law are investigated. For both cases, dispersion equations are derived, which are solved numerically. Asymptotics of the roots of dispersion equations for small and large frequencies are also obtained. The analysis of the obtained solutions made it possible to draw conclusions about the influence of hereditary factors on the behavior of dispersion curves. A comparative analysis of numerical solutions and their asymptotics is carried out.
\end{abstract}

Keywords: Dispersion Equations, Stress-Strain State, Hereditarily Elastic Layer, Asymptotics

\section{Introduction}

The increasing need to reduce the vibrations of structural elements caused by loads with a broadband frequency spectrum (for example, aircraft body vibrations) has drawn attention to viscoelastic coatings as a possible solution to this problem. The frequency equation for such systems was obtained from the theory of elasticity, for example [1-3]. In [1] the problem of the propagation of waves of three-layer elastic beams led to a transcendental equation containing hyperbolic functions. The solution of the corresponding transcendental equation for the plates was obtained only for the two lower branches $[2,3]$. The calculation of the lower branches was performed using the expansion of transcendental functions into power series, which limits the range of applicability of the results. Another type of solution was obtained in the problem of longitudinal oscillations of a cylindrical rod with a viscoelastic coating [4]. The propagation of bending waves in a plate with viscoelastic coatings in a simplified formulation is considered in $[5,6]$.

It is known that most of the information on the behavior of the waveguide is provided by the dispersion equation. Numerical analysis of the dispersion equations obtained during the investigation of the propagation of harmonic waves in a hereditarily elastic plate with two viscoelastic coatings is performed.

Taking into account the rheological properties of the material is accompanied by dispersion of the waves. The mechanisms by which the energy of elastic waves is converted into heat are not entirely clear. Different loss mechanisms are proposed $[7,8,9]$, but not one of them does not fully meet all the requirements. Probably the most important mechanisms are internal friction in the form of sliding friction (or sticking, and then slipping) and viscous losses in pore fluids; the latter mechanism is most significant in strongly permeable rocks. Other effects that are probably generally less significant are the loss of some of the heat generated in the phase of compression of wave motion by thermal conductivity, piezoelectric and thermoelectric effects 
and the energy going to the formation of new surfaces (which plays an important role only near the source).

Formulation of the problem.

We consider the propagation of harmonic waves in an infinite hereditarily elastic layered body, bounded by the planes $\mathrm{z}= \pm \mathrm{h}$ in the Cartesian coordinate system (Figure 1). The Oyz plane is compatible with the middle surface of the layer. We shall consider the propagation of waves in the direction of the $\mathrm{x}$ axis. Dynamic layer VAT will be described by the equations of motion for the case of a plane problem

$$
\begin{gathered}
\frac{\partial \sigma_{11}^{(n)}}{\partial x}+\frac{\partial \sigma_{13}^{(n)}}{\partial z}=\rho_{n} \frac{\partial^{2} \vartheta_{1}^{(n)}}{\partial t^{2}} ; \\
\frac{\partial \sigma_{31}^{(n)}}{\partial x}+\frac{\partial \sigma_{33}^{(n)}}{\partial z}=\rho_{n} \frac{\partial^{2} \vartheta_{3}^{(n)}}{\partial t^{2}},(\mathrm{n}=1,2,3)
\end{gathered}
$$

and the equations of state for hereditarily elastic material.

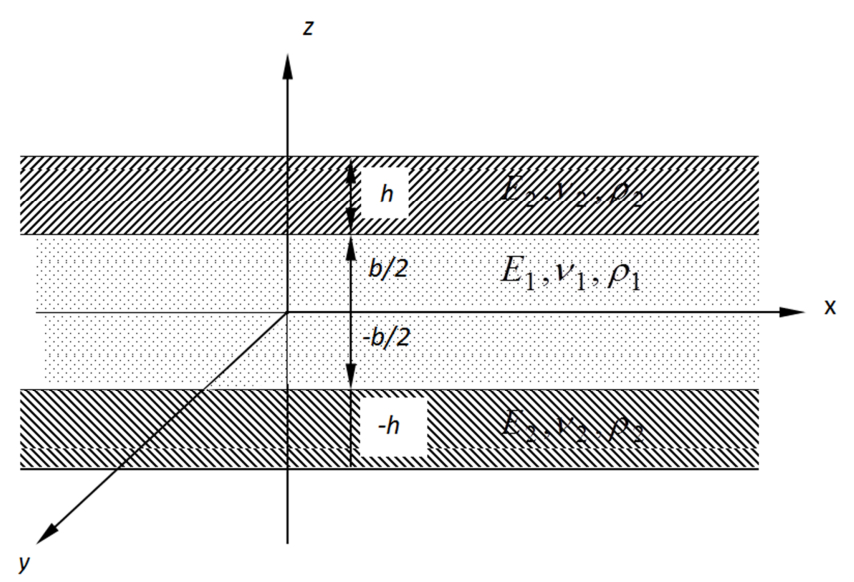

Figure 1. The design scheme.

In the present paper we take the equations of state in an integral operator form (the Rabotnov kernel [10])

$$
\begin{gathered}
\tilde{E}_{n} \frac{\partial \vartheta_{1}^{(n)}}{\partial x}=\sigma_{11}^{(n)}-\tilde{v}_{n}\left(\sigma_{22}^{(n)}+\sigma_{33}^{(n)}\right) ; \\
\tilde{E}_{n} \frac{\partial \vartheta_{3}^{(n)}}{\partial z}=\sigma_{33}^{(n)}-\tilde{v}_{n}\left(\sigma_{11}^{(n)}+\sigma_{22}^{(n)}\right) \\
0=\sigma_{22}^{(n)}-\tilde{v}_{n}\left(\sigma_{11}^{(n)}+\sigma_{33}^{(n)}\right) ; \\
\frac{1}{2} \tilde{E}_{n}\left(\frac{\partial \vartheta_{1}^{(n)}}{\partial z}+\frac{\partial \vartheta_{3}^{(n)}}{\partial x}\right)=\left(1+\tilde{v}_{n}\right) \sigma_{13}^{(n)}
\end{gathered}
$$

In (1) and (2) the following notation is used: $\sigma_{i j}^{(n)}$ components of the stress tensor, $\vartheta_{i}^{(n)}$ - displacement vector components,

$$
\tilde{E}_{n}=E_{n}\left(1-\Gamma_{n}^{\bullet}\right) ; \quad \tilde{v}_{n}=v_{n}+\frac{1-2 v_{n}}{2} \Gamma_{n}^{\bullet}
$$

$$
\Gamma_{n}^{\bullet} f(t)=m_{n} \int_{-\infty}^{t} \ni_{-1 / 2}^{(n)}\left(-\beta_{n}, t-\tau\right) f(\tau) d \tau
$$

Here E, v- are the instantaneous Young's modulus and Poisson's ratio, $\mathrm{m}_{\mathrm{n}}, \beta_{\mathrm{n}}$ - parameters of the material. As the kernel of the integral operator we use the fractional exponential function of Rabotnov [12]

$$
m_{n} \ni_{-1 / 2}^{(n)}(-\beta, t)=m_{n} t^{-1 / 2} \sum_{j=0}^{\infty} \frac{\left(-\beta_{n}\right)^{j} t^{j / 2}}{\Gamma[(j+1) / 2]}
$$

where $\Gamma(\mathrm{j})=\int_{0}^{\infty} y^{j-1} \exp (-y) d y$ - gamma function.

In the case when the Koltunov-Rzhanitsen relaxation [1922] core is used in place (3), then the elastic modulus is replaced by the operators, i.e. $\tilde{E}_{n}-$ the elastic modulus of elasticity has the form $[4,5]$ :

$$
\tilde{E}_{n} \phi(t)=E_{0 n}\left[\phi(t)-\int_{0}^{t} R_{E n}(t-\tau) \phi(t) d \tau\right]
$$

$\phi(t)$ - arbitrary time function; $R_{E n}(t-\tau)$ - relaxation core; $E_{01}$ - instantaneous modulus of elasticity; We assume the integral terms in (4) to be small, then the functions $\phi(t)=\psi(t) e^{-i \omega_{R} t}$, where $\psi(t)$ - a slowly varying function of time, $\omega_{R}$ - real constant. Then [13], we replace (4) by approximations of the form

$$
\bar{E}_{n} \phi=E_{0 j}\left[1-\Gamma_{j}^{C}\left(\omega_{R}\right)-i \Gamma_{j}^{S}\left(\omega_{R}\right)\right] \phi
$$

where $\Gamma_{n}{ }^{C}\left(\omega_{R}\right)=\int_{0}^{\infty} R_{n}(\tau) \cos \omega_{R} \tau d \tau, \Gamma_{n}{ }^{S}\left(\omega_{R}\right)=\int_{0}^{\infty} R_{n}(\tau) \sin \omega_{R} \tau d \tau$, respectively, the cosine and sine Fourier images of the relaxation core of the material. As an example of a viscoelastic material, we take three parametric relaxation nuclei $R_{n}(t)=A_{n} e^{-\beta_{n} t} / t^{1-\alpha_{j n}}$. On the influence function $R_{n}(t-\tau)$ The usual requirements of integrability, continuity (except for), signs - definiteness and monotonicity are imposed:

$$
R>0, \frac{d R(t)}{d t} \leq 0,0<\int_{0}^{\infty} R(t) d t<1 .
$$

In the study of natural oscillations, we will investigate the properties of those modes (the modes are understood to mean particular solutions of the equations of motion in displacements that satisfy homogeneous boundary conditions on the face surfaces), which vary in time according to the harmonic law and satisfy the equations of motion (1), equations of state (2) and homogeneous boundary conditions on the faces: 
- in the case of a rigid contact at the interface, the condition of continuity of the corresponding components of the stress tensor and

vector of displacement, i.e.

$$
\sigma_{z z}^{(1)}=\sigma_{z z}^{(2)} ; \quad \sigma_{x z}^{(1)}=\sigma_{x z}^{(2)} ; \quad \vartheta_{1}^{(1)}=\vartheta_{1}^{(2)} ; \quad \vartheta_{3}^{(1)}=\vartheta_{3}^{(2)} .
$$

- If there is no friction at the interface,

$$
\sigma_{z z}^{(1)}=\sigma_{z z}^{(2)} ; \quad \sigma_{x z}^{(1)}=\sigma_{x z}^{(2)}=0 ; \quad \vartheta_{3}^{(1)}=\vartheta_{3}^{(2)} ;
$$

- On the free surface, the condition of freedom from stress is set, i.e.

$$
\sigma_{z z}^{(1)}=0 ; \quad \sigma_{z x}^{(1)}=0
$$

where

$$
\begin{gathered}
\sigma_{x x}^{(n)}=\lambda_{n} \theta_{n}+2 \mu_{n} \frac{\partial \vartheta_{1}^{(n)}}{\partial x} ; \sigma_{x z}^{(j)}=\mu_{j}\left(\frac{\partial \vartheta_{1}^{(n)}}{\partial z}+\frac{\partial \vartheta_{3}^{(n)}}{\partial x}\right) \\
\sigma_{z z}^{(j)}=\lambda_{j} \theta_{j}+2 \mu_{j} \frac{\partial \vartheta_{3}^{(n)}}{\partial z} \theta_{j}=\frac{\partial \vartheta_{1}^{(n)}}{\partial x}+\frac{\partial \vartheta_{3}^{(n)}}{\partial z} .
\end{gathered}
$$

Thus, taking (5) into account, we arrive at the following system of equations:

$$
\begin{gathered}
\frac{\partial \sigma_{11}^{(n)}}{\partial x}+\frac{\partial \sigma_{13}^{(n)}}{\partial z}=\rho_{n} \frac{\partial^{2} \vartheta_{1}^{(n)}}{\partial t^{2}} ; \\
\frac{\partial \sigma_{31}^{(n)}}{\partial x}+\frac{\partial \sigma_{33}^{(n)}}{\partial z}=\rho_{n} \frac{\partial^{2} \vartheta_{3}^{(n)}}{\partial t^{2}} \\
\bar{E}_{n} \frac{\partial \vartheta_{1}^{(n)}}{\partial x}=\sigma_{11}^{(n)}-v_{n}\left(\sigma_{22}^{(n)}+\sigma_{33}^{(n)}\right) \\
\bar{E}_{n} \frac{\partial \vartheta_{3}^{(n)}}{\partial z}=\sigma_{33}^{(n)}-v_{n}\left(\sigma_{11}^{(n)}+\sigma_{22}^{(n)}\right) \\
0=\sigma_{22}^{(n)}-v_{n}\left(\sigma_{11}^{(n)}+\sigma_{33}^{(n)}\right) \\
\frac{1}{2} \bar{E}_{n}\left(\frac{\partial \vartheta_{1}^{(n)}}{\partial z}+\frac{\partial \vartheta_{3}^{(n)}}{\partial x}\right)=\left(1+v_{n}\right) \sigma_{13}^{(n)}
\end{gathered}
$$

with boundary conditions (6).

\section{Methods of Solution}

The solution of the system of differential equations (2) (6) (or (7) and (6)) for $\sigma_{i j}^{(n)}$ - components of the stress tensor and $\vartheta_{i}^{(n)}$ — the components of the displacement vector will be sought in the form

$$
\begin{gathered}
\sigma_{i j}^{(n)}=\sigma_{i j}^{n}(\mathrm{z}) \exp (\mathrm{i} \omega \mathrm{t}-\mathrm{ikx}), \\
\vartheta_{j}^{n}=\mathrm{V}_{j}^{n}(\mathrm{z}) \exp (\mathrm{i} \omega \mathrm{t}-\mathrm{ikx}),
\end{gathered}
$$

where $\sigma_{i j}^{n}(\mathrm{z})$ и $\mathrm{V}_{j}^{n}(\mathrm{z})$ - amplitude complex vector function $(\mathrm{j}=1,2) ; k\left(k=k_{R}+i k_{I}\right)$ - wave number; $C\left(C=C_{R}\right.$ $\left.+i C_{i}\right)$ - complex phase velocity; $\omega$ - complex frequency.

To clarify their physical meaning, consider two cases:

1) $k=k_{R} ; C=C_{R}+i C_{i}$, then the solution (8) has the form of a sinusoid with respect to $\mathrm{x}$, the amplitude of which decays in time;

2) $k=k_{R}+i k_{l} ; C=C_{R}$, Then at each point $\mathrm{x}$ the oscillations are steady, but with respect to $\mathrm{x}$ they decay.

In both cases, the imaginary parts $\mathrm{k}_{\mathrm{I}}$ or $\mathrm{C}_{\mathrm{I}}$ characterize the intensity of dissipative processes. Substituting relations (8) into a system of partial differential equations (2), we obtain a system of ordinary differential equations of the first order, solved with respect to the derivatives:

$$
\begin{gathered}
\frac{d \sigma_{13}^{n}}{d z}=E_{4 n} \sigma_{33}^{n}-E_{5 n} V_{1}^{n} ; \\
\frac{d \sigma_{33}^{n}}{d z}=\delta_{k} \sigma_{31}^{n}-\Omega_{n}^{2} V_{3}^{n} ; \\
\frac{d V_{3}^{n}}{d z}=E_{1 n} \sigma_{33}^{n}-E_{2 n} V_{1}^{n} ; \\
\frac{d V_{1}^{n}}{d z}=E_{3 n} \sigma_{13}^{n}+\delta_{k} V_{3}^{n} ; \\
\sigma_{11}^{n}=\frac{v_{n}^{F}}{1-v_{n}^{F}} \sigma_{33}^{n}-\frac{E_{n} E_{n}^{F}(\delta+i k ;)}{1-\left(v_{n}^{F}\right)^{2}} V_{1}^{n} ; \\
\sigma_{22}^{n}=v_{n}^{F}\left(\sigma_{11}^{n}+\sigma_{33}^{n}\right) .
\end{gathered}
$$

where

$$
\begin{aligned}
& E_{1 n}=\frac{1}{E_{n} E_{n}^{F}}\left(1-\left(v_{n}^{F}\right)^{2}-\frac{\left(v_{n}^{F}\right)^{2}\left(1+v_{n}^{F}\right)}{1-v_{n}^{F}}\right) ; E_{2 n}=\frac{\delta_{k}}{1-\left(v_{n}^{F}\right)^{2}} ; \delta_{k}=\delta+i k ; \\
& E_{3 n}=\frac{2\left(1+v_{n}^{F}\right)}{E_{n} E_{n}^{F}} ; E_{4 n}=\frac{v_{n}^{F}(\delta+i k)}{1-v_{n}^{F}} ; E_{5 n}=\frac{E_{n} E_{n}^{F}(\delta+i k)^{2}}{1-\left(v_{n}^{F}\right)^{2}}+\rho_{n} \omega^{2} ; \Omega_{n}=\rho_{n} \omega^{2} . \\
& E_{n}^{F}=1-\frac{m_{n}}{\beta_{n}+\sqrt{i \omega}} ; v_{n}^{F}=v_{n}+\frac{1-2 v_{n}}{2} \frac{m_{n}}{\beta_{n}+\sqrt{i \omega}} .
\end{aligned}
$$

At the interface $\left(\mathrm{z}=\mathrm{h}_{\mathrm{n}}\right)$ (in the case of a rigid contact), the condition of continuity of the corresponding components of the stress tensor and the displacement vector is set, i.e.

$$
\begin{array}{ll}
\sigma_{z z}^{n}\left(h_{n}\right)=\sigma_{z z}^{n+1}\left(h_{n}\right) ; & \sigma_{x z}^{n}\left(h_{n}\right)=\sigma_{x z}^{n+1}\left(h_{n}\right) ; \\
V_{1}^{n}\left(h_{n}\right)=V_{1}^{n+1}\left(h_{n}\right) ; & V_{3}^{n}\left(h_{n}\right)=V_{3}^{n+1}\left(h_{n}\right) .
\end{array}
$$

- if at the interface $\left(z=h_{n}\right)$ there is no friction, then

$$
\sigma_{z z}^{1}=\sigma_{z z}^{2} ; \quad \sigma_{x z}^{1}=\sigma_{x z}^{2}=0 ; \quad V_{3}^{1}=V_{3}^{2} ;
$$

- on a free surface $\left(\mathrm{z}=\mathrm{h}_{\mathrm{k}}\right)$ the condition of freedom from stresses is set, i.e.

$$
\sigma_{z z}^{1}=0 ; \quad \sigma_{z x}^{1}=0
$$

Thus, the spectral boundary value problem (9) - (10) is 
formulated with respect to the parameter $\omega$, which describes the propagation of waves in three-layer bodies. The posed spectral problem (7) - (8) in dimensionless variables is solved by the method of orthogonal sweep with a combination of the Muller method [23] on complex arithmetic:

$$
\xi_{n}=\frac{x}{h_{n}} ; \quad \zeta_{n}=\frac{z}{h_{n}} ; \quad t^{n}=\frac{c_{2 n} t}{h_{n}}, c_{2 n}=\sqrt{\frac{E_{n}}{2\left(1+v_{n}\right) \rho_{n}}}
$$

\subsection{Task 1}

Consider a plate in which the neutral plane coincides with the plane $\mathrm{x}, \mathrm{z}$, and $\mathrm{y}$ coordinate in the direction of thickness, in the $\mathrm{x}$ direction, the plate is propagated by harmonic bending waves (Figure 1). The main material of the plate occupies the region $-\frac{b}{2} \leq z \leq \frac{b}{2}$, and for the covering, each of which have everywhere the same thickness $h$, occupies the region $\left(\frac{b}{2} \leq z \leq h,-h \leq z \leq-\frac{b}{2}\right)$. Let $\mathrm{B}_{l}, \quad \mu_{1}$ and $\rho_{1}$ respectively, the bulk modulus, the shear modulus, and the density of the plate material. Then the solution of the Rayleigh-Lembe problem for the base layer is obtained by methods of separating the variables and determining the displacements $u, \mathrm{v}, w$ (in the $\mathrm{x}, \mathrm{y}$ and $\mathrm{z}$ directions, respectively) in the propagation of a harmonic wave with a frequency $\omega$ and a wave number $\mathrm{k}$ have the form [17]

$$
\begin{gathered}
u=0, \\
\vartheta=\left(\alpha A_{1} \operatorname{ch} \alpha z-k C_{1} \operatorname{sh} \beta z\right) e^{i(\omega t-k x),}, \\
w=\left(-i k A_{1} \operatorname{ch} \alpha z+i \beta C_{1} \operatorname{sh} \alpha z\right) e^{i(\omega t-k x),},
\end{gathered}
$$

for $-\frac{b}{2} \leq y \leq \frac{b}{2}$, where $A_{1}$ and $\mathrm{C}_{1}$ - constants, and the parameter $\alpha_{1}$ and $\beta_{1}$ are defined by expressions

$$
\alpha^{2}=k^{2}-\frac{\omega^{2} \rho_{1}}{B_{1}+\frac{4}{3} \mu_{1}} ; \beta^{2}=k^{2}-\frac{\omega^{2} \rho_{1}}{\mu_{1}} .
$$

On the basis of the ratios of the linear theory of elasticity between stresses and deformations of a homogeneous isotropic material, the plate has view

$$
\begin{aligned}
& \sigma_{x z}=(G-2 / 3 \mu) A_{1}\left(\alpha^{2}-k^{2}\right) \operatorname{sh} \alpha z \\
& \sigma_{y y}=A_{1}\left[(G-2 / 3 \mu)\left(\alpha^{2}-k^{2}\right)+2 \mu \alpha^{2}\right] \operatorname{sh} \alpha z-2 \mu k \beta C_{1} \operatorname{sh} \beta z \\
& \sigma_{x y}=\sigma_{x x}=0 \\
& \sigma_{y z}=-2 \mu i k A_{1} \alpha \operatorname{ch} x z+i \mu C_{1}\left(k^{2}+\beta^{2}\right) \operatorname{ch} \beta z \\
& \sigma_{y y}=A_{1}\left[(G+2 / 3 \mu)\left(\alpha^{2}-k^{2}\right)+2 \mu \alpha^{2}\right] \operatorname{sh} \alpha z+2 \mu k \beta C_{1} \operatorname{sh} \beta z
\end{aligned}
$$

The expression for displacements of the points of the upper outer covering, satisfying the equations of motion [1], generally have the form:

$$
\begin{aligned}
& u=0 ; \\
& \vartheta=-\alpha c\left[\beta_{1} \operatorname{sh} \alpha_{p}(z-H)+F_{1} \operatorname{ch} \alpha_{p}(z-H)\right]+ \\
& +k\left[D_{1} \operatorname{ch} \beta_{p}(z-H)+M_{1} \operatorname{sh} \beta_{p}(z-H)\right] ; \\
& w=i k\left[\beta_{1} \operatorname{ch} \alpha_{p}(z-H)+F_{1} \operatorname{sh} \alpha_{p}(z-H)\right]- \\
& -i \beta_{c}\left[D_{1} \operatorname{sh} \beta_{p}(z-H)+M_{1} \operatorname{ch} \beta_{p}(z-H)\right] ;
\end{aligned}
$$

Here $\mathrm{B}_{1}, \mathrm{D}_{1}, \mathrm{~F}_{1}, \mathrm{M}_{1}-$ constant, and the parameters $\alpha_{p}$ and $\beta_{p}$ is determined by analogy with expressions (11) by the following dependences

$$
\alpha_{p}^{2}=k^{2}-\frac{\omega^{2} \rho_{p}}{G_{p}+\frac{4}{3} \mu_{p}} ; \beta_{p}^{2}=k^{2}-\frac{\omega^{2} \rho_{p}}{\mu_{p}}
$$

where the subscript $\mathrm{c}$ refers to the characteristics of the coating material. For the region $-H=-(h+b / 2) \leq z \leq-b / 2$ it is possible to measure an analogous family of dependencies using the antisymmetric properties of the flexural wave

$$
u(z)=u(-z) ; \vartheta(z)=\vartheta(-z) ; w(z)=-w(-z)
$$

Further, using properties (12) and restricting ourselves to the investigation of the domain $0 \leq z \leq H$. On the free surface $\mathrm{z}=\mathrm{H}$, the following boundary conditions must be satisfied:

$$
\sigma_{x z}=\sigma_{z z}=\sigma_{x z}=0
$$

In addition, on the surface of the contacts $\mathrm{z}=\mathrm{b}$, the continuity conditions of displacements must be satisfied

$$
u\left(b^{-}\right)=u\left(b^{+}\right) ; \vartheta\left(b^{-}\right)=\vartheta\left(b^{+}\right), w\left(b^{-}\right)=w\left(b^{+}\right)
$$

and the conditions of equality

$$
\sigma_{x z}\left(b^{-}\right)=\sigma_{x z}\left(b^{+}\right) ; \sigma_{z z}\left(b^{-}\right)=\sigma_{z z}\left(b^{+}\right) ; \sigma_{y z}\left(b^{-}\right)=\sigma_{y z}\left(b^{+}\right) .
$$

On the basis of conditions (11), (12), (13) and the symmetry conditions, we form a system of six linear homogeneous algebraic equations with respect to unknown constant $A_{1}, \mathrm{~B}_{1}, \mathrm{C}_{1}, \mathrm{D}_{1}, \mathrm{~F}_{1}$ and $\mathrm{M}_{1}$; the first two of these constants determine the deformed state of the plate, the other four - the deformed state of the upper layer of the coating. As a result, we obtain systems of homogeneous algebraic equations with complex coefficients $[C]\{q\}=\{0\}$. A system of homogeneous algebraic equations has a nontrivial solution, under the condition that the determinant of the basic matrix

$$
[C]=0
$$


where

$$
\begin{aligned}
& C_{11}=\alpha \operatorname{ch} \alpha k ; C_{12}=-k \operatorname{ch} \beta k ; C_{13}=\alpha_{c} \operatorname{sh} \alpha_{c}(b-H) ; \\
& C_{14}=\alpha_{c} \operatorname{ch} \alpha_{c}(b-H) ; C_{15}=k \operatorname{ch} \beta_{c}(b-H) ; \\
& C_{16}=-k \operatorname{sh} \beta(b-H) ; C_{21}=-k \operatorname{sh} \alpha b ; C_{22}=\beta \operatorname{sh} \beta h \\
& C_{23}=-k \operatorname{ch} \alpha_{c}(b-H) ; C_{24}=-k c h \alpha_{c}(b-H) ; \\
& C_{25}=\beta_{c} \operatorname{sh} \beta_{c}(b-H) ; C_{26}=\beta \operatorname{sh} \beta_{c}(b-H) ; \\
& C_{31}=\alpha_{1} \operatorname{sh} \alpha b ; C_{32}=-2 \mu k \beta \operatorname{sh} \beta b \\
& C_{33}=-\alpha_{2} \operatorname{ch} \alpha_{c}(b-H) ; C_{34}=-\alpha_{2} \operatorname{ch} \alpha_{c}(b-H) ; \\
& C_{35}=2 \mu_{c} k \beta \beta_{c} \operatorname{sh} \beta_{c}(b-H) ; C_{35}=2 \mu_{c} k \beta_{c} \operatorname{ch} \beta_{c}(b-H) ; \\
& C_{41}=-2 \mu k \alpha_{c h} \alpha h ; C_{42}=\mu \alpha_{3} \operatorname{ch} \beta h ; \\
& C_{43}=-2 \mu_{c} k \alpha_{c} s h \alpha_{c}(b-H) ; C_{44}=-2 \mu_{c} k \alpha_{c} \operatorname{ch} \alpha_{c}(b-H) ; \\
& C_{45}=\mu_{c} \alpha_{1} \operatorname{ch} \beta_{c}(b-H) ; C_{46}=\mu_{c} \alpha_{1} \operatorname{sh} \beta_{c}(b-H) ; \\
& C_{51}=C_{52}=C_{54}=C_{55}=0 ; C_{53}=-\alpha_{3} ; C_{56}=-2 \mu_{c} k \beta_{c} ; \\
& C_{61}=C_{62}=C_{63}=C_{66}=0 ; C_{64}=-2 \mu_{c} k \alpha_{c} ; C_{65}=\mu_{c} \alpha_{c} ;
\end{aligned}
$$

where

$$
\begin{aligned}
& \alpha_{1}=G\left(k^{2}-\alpha^{2}\right)-\frac{2}{3} \mu\left(k^{2}+\alpha^{2}\right) ; \alpha_{2}=k^{2}+\alpha^{2} \\
& \alpha_{3}=G\left(k^{2}-\alpha_{c}^{2}\right)-\frac{2}{3} \mu_{c}\left(k^{2}+2 \alpha_{c}^{2}\right) ; \alpha_{4}=k^{2}+\beta_{c}^{2} .
\end{aligned}
$$

The transcendental equation (14) can be solved numerically by the method of Muller [7,23].

\subsection{Task 2. The Propagation of Waves in a Viscoelastic Three-Layer Body on a Rigid Base}

Suppose that in a Cartesian coordinate system a sequence of parallel planes is given. Suppose that the spaces between planes are filled with isotropic elastic media forming parallel layers. In the theoretical study of the described processes, we will assume that within each layer the wave propagation is described by the equations of elasticity theory (Lame). On the boundary of two bodies, the conditions of a rigid or sliding contact are set. With rigid contact at the interface, the condition of continuity of the corresponding components of the stress tensor and the displacement vector is established. On the free surface, the condition of freedom from stress is set. The problem is solved in the displacement potentials. The Sommerfeld radiation conditions satisfy the potential function at infinity. The dispersion equation has the form:

$$
\left|\beta_{i j}\right|=0, \quad i, j=1,2, \ldots, 12
$$

where $\beta_{1,1}=\left(1+\bar{s}_{1}^{2}\right) \exp \left(-k q_{1} h\right)^{*} ; \quad \beta_{1,2}=\left(1+\bar{s}_{1}^{2}\right) \exp \left(-k q_{1} h_{1}^{*}\right)$

$$
\begin{aligned}
& \beta_{1,3}=-2 \exp \left(-k s_{1} h\right)^{*} ; \quad \beta_{14}=2 \exp \left(k s_{1} h^{*}\right) ; \\
& \beta_{1,5}=\beta_{1,6}=0 ; \quad \beta_{1,7}=\beta_{1,8}=0 ; \quad \beta_{1,9}=\beta_{1,10}=\beta_{1,11}=\beta_{1,12}=0 ; \\
& \beta_{2,1}=-2 \bar{q}_{1} \exp \left(-k q_{1} h_{1}^{*}\right) ; \quad \beta_{2,2}=2 \bar{q}_{1} \exp \left(k q_{1} h_{1}^{*}\right) ; \\
& \beta_{2,5}=\beta_{2,6}=\beta_{2,7}=\beta_{2,8}=0 ; \quad \beta_{2,9}=\beta_{2,10}=\beta_{2,11}=\beta_{2,12}=0 \\
& \beta_{2,3}=\left(\overline{s_{1}}+\frac{1}{\bar{s}_{1}}\right) \times \exp \left(-k \overline{s_{1}} h\right) ; \quad \beta_{2,4}=\left(\overline{s_{1}}+\frac{1}{\bar{s}_{1}}\right) \times \exp \left(k \bar{s}_{1} h\right) ; \\
& \beta_{3,1}=\beta_{3,2}=\beta_{3,3}=\beta_{3,4}=0 ; \quad \beta_{3,5}=\beta_{3,6}=\beta_{3,7}=\beta_{3,8}=0 ; \\
& \beta_{3,9}=\left(1+\bar{s}_{2}^{2}\right) \exp \left(k q_{2} h_{2}\right) ; \quad \beta_{3,10}=\left(1+\bar{s}_{2}^{2}\right) \times \exp \left(-k q_{2} h_{2}\right) ; \\
& \beta_{3,11}=-2 \exp \left(k \bar{s}_{21} h_{2}\right) ; \quad \beta_{3,12}=2 \exp \left(k \bar{s}_{21} h_{2}\right) ; \\
& \beta_{4,1}=\beta_{4,2}=\beta_{4,3}=\beta_{4,4}=0 ; \beta_{4,5}=\beta_{4,6}=\beta_{4,7}=\beta_{4,8}=0 ; \\
& \beta_{4,9}=-2 \bar{q}_{2} \exp \left(k \bar{q}_{2} h_{2}\right) ; \quad \beta_{4,10}=2 \bar{q}_{2} \exp \left(-k \bar{q}_{2} h_{2}\right) ; \\
& \beta_{4,11}=\left(\bar{s}_{2}+\bar{s}_{2}^{-1}\right) \exp \left(k \bar{s}_{2} h_{2}\right) ; \quad \beta_{4,12}=\left(\bar{s}_{2}+\bar{s}_{2}^{-1}\right) \exp \left(-k \bar{s}_{2} h_{2}\right)
\end{aligned}
$$

The remaining elements (15) of the determinant are written in a similar way $h_{1}^{*}=h_{1}+h_{2}$. Now this equation will be solved with respect to $\omega / \mathrm{k}$ for different values of $\mathrm{k}$. The roots of the equation are calculated on a computer with the following values of the dimensionless parameters:

$$
\begin{aligned}
& C^{2}{ }_{L 1}=0.622 ; C^{2}{ }_{L 2}=3.360 ; C^{2}{ }_{23}=3.360 ; C^{2}{ }_{S 1}=0.776 ; \\
& C^{2}{ }_{S 2}=1.230 ; \\
& C^{2}{ }_{S 3}=3.000 ; \mu_{1}=0.170 ; \mu_{2}=0.30 ; \mu_{3}=0.3
\end{aligned}
$$

\section{Results}

The results are plotted as dependency curves $(\xi=\mathrm{kH}, \mathrm{H}=$ $\mathrm{h}_{1}+\mathrm{h}_{2}$ ) from $\omega$ for $\mathrm{h}^{*}{ }_{1} / \mathrm{H}=0.5$. Let's consider two variants of the system. In the first variant, a homogeneous system is considered (Figure 4). coefficient behavior $\delta$ radically changed: dependence $\omega_{\mathrm{I}} \sim \xi$ became nonmonotonic. Of particular interest for practice is the minimum for fixing $\xi$ value of the damping coefficient, the value of $\delta$ determines the damping properties as a whole.

In the case of a homogeneous system, $\delta$ is determined by the imaginary part of the complex natural frequency modulo the first. In the case of an inhomogeneous system $\left(\mathrm{R}_{\mathrm{E} 2}=0\right)$ The role of the global damping coefficient is imaginary parts of both the first and second frequencies (Figure 5). The mathematical and physical aspects of this effect are explained in $[13,14,15,16]$. The change in the parameter, on which the global coefficient of damping depends so substantially, can be achieved by varying the geometric dimensions or physical properties, thereby opening up the perspective possibility of effectively controlling the damping characteristics of dissipatively inhomogeneous viscoelastic systems.

The frequency equation (14) is solved by the Mueller method. In all cases, the Poisson's ratio of the middle layer is taken as 0.25 ; attitudes $\rho_{1} / \rho$ densities of the coating materials and the middle layer are 0.35 , and the ratio $G_{1} / \mu$ module of the volume compression of the coating material to the shear modulus of the material of the middle layer 0.20 . The values given correspond to a system that consists of an elastic aluminum middle layer and a coating, is made of a typical 
high-polymer material, and it is proposed that purely volumetric deformation of the coating during vibrations is not accompanied by energy dissipation. Various values of $h /$ $b$ were considered - the ratio of the thickness of the coating to the half-thickness of the middle layer and the different values of the coefficients $\gamma$ - of the energy dissipation in the material under shear deformations The results are presented in the form of graphs of the dependences of the real part $\Omega\left(\omega^{2}\right)$ and the coefficient of loss from the dimensionless wave number $h / x=k h / 2 \pi$ (the ratio of the half-thickness of the middle layer to the wavelength). The absolute error in calculating the eigenvalues (with respect to the exact equations) lies in the limit $14 \cdot 10^{-6}$. Figure 2 shows the change in the real and imaginary parts of the complex frequency as a function of the dimensionless wavelength. $\gamma=$ 0,35 and the relative thickness of the coating $h / \lambda=0,1$.

In the case of a dissipative homogeneous system $\delta$ (let's call it the global damping coefficient) is entirely determined by the imaginary part. First modulo complex natural frequency

$$
\delta=\min \left(\Omega_{i k}\right), k=1,2, \ldots n .
$$

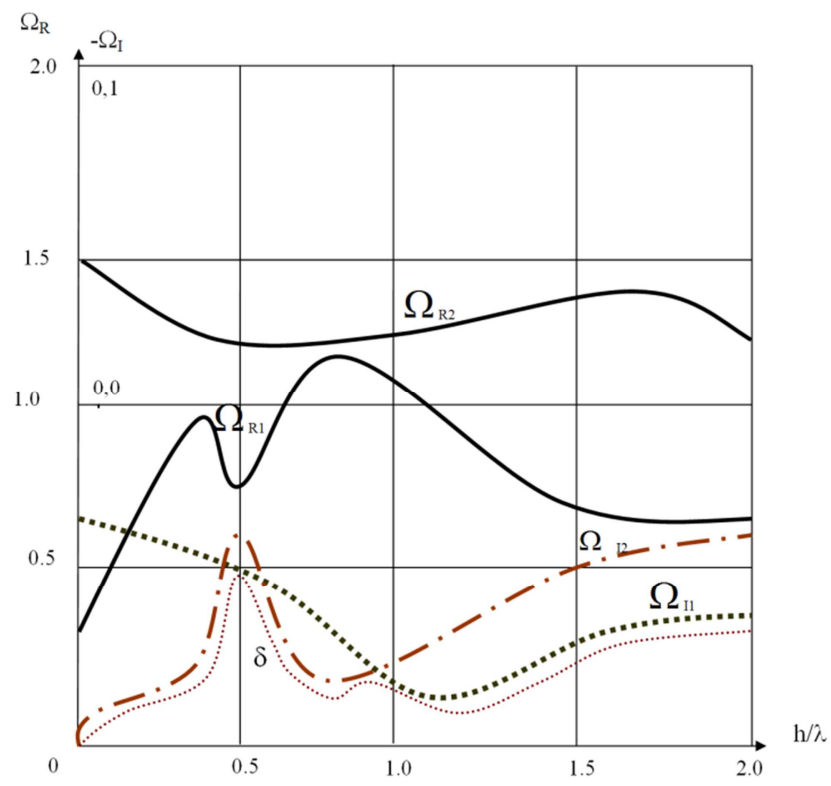

Figure 2. Dependence of natural frequencies $\left(\Omega_{R}\right)$ and the damping coefficients of the wavelength.

In the case of dissipatively inhomogeneous mechanical systems [5, 6], a nonmonotonic dependence of the damping coefficient on the wavelength is observed. The role of the global damping coefficient is played here by the imaginary parts of the first and second eigenfrequencies. When the corresponding frequencies approach $\left(\Omega_{\mathrm{Rk}}\right)$ intersection of the imaginary parts of the first and second, the modes of the natural frequencies. A similar effect was observed when using the fractional-exponential kernel of the heredity Rabotnov Yu.N [24,25]. A similar effect was found in studies of systems with a finite number of degrees of freedom.

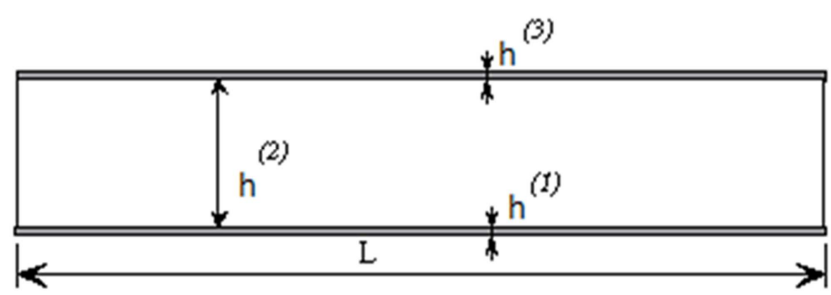

$\omega_{4}=534,7 \mathrm{~Hz}$

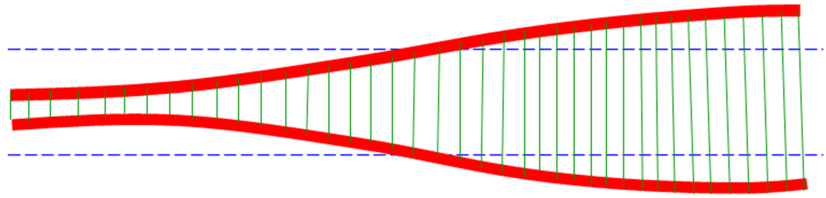

$\omega_{5}=620,2 \mathrm{~Hz}$

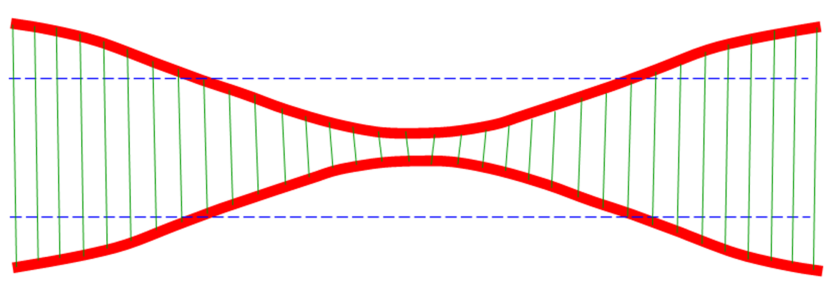

$\omega_{6}=711,1 \mathrm{~Hz}$

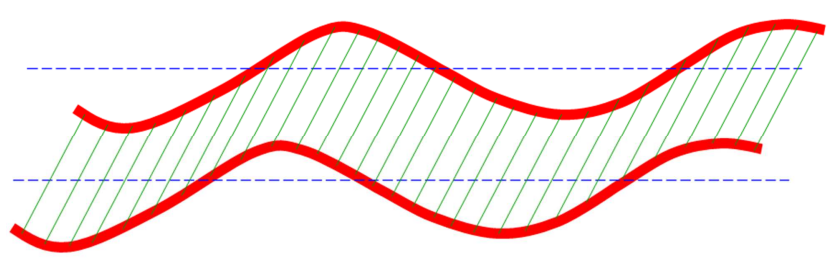

$\omega_{7}=732,6 \mathrm{~Hz}$

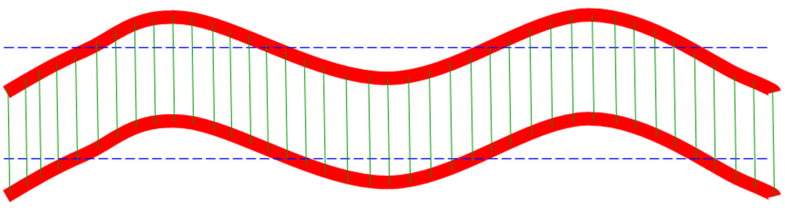

$\omega_{1}=116,5 \mathrm{~Hz}$

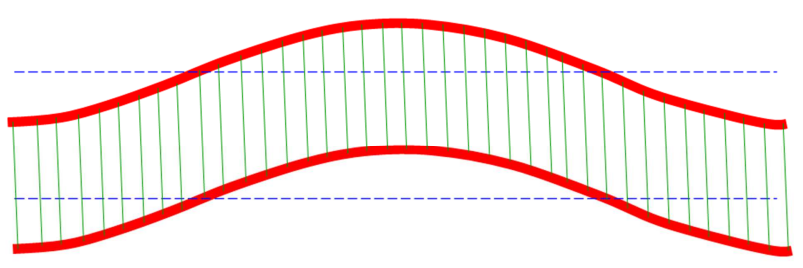

$\omega_{2}=121,3 \mathrm{~Hz}$

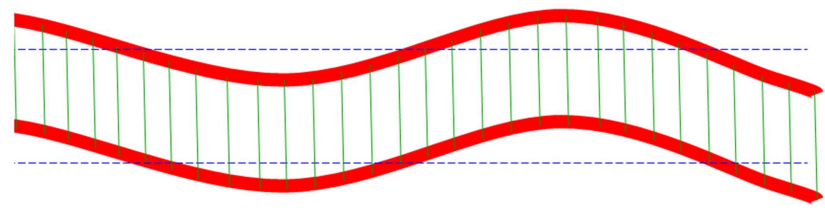

$\omega_{3}=327,9 \mathrm{~Hz}$ 


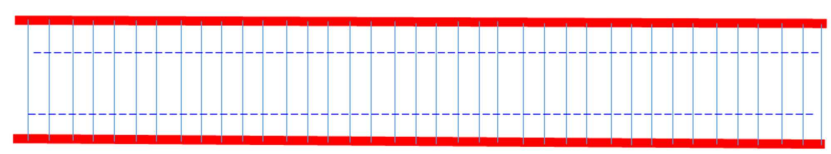

Figure 3. Forms of plate vibrations at different frequencies.

Figure 3 shows the change in the shape of the plate oscillations at different frequencies for $\mathrm{h}=0,01 \mathrm{~m}$, $E_{2}=5,5.10^{8} \mathrm{~Pa}, \quad E_{1}=3,5.10^{10} \mathrm{~Pa}, \quad b=0.08 \mathrm{~m}, \quad \mathrm{~L}=1 \mathrm{~m}$, $\rho_{1}=27 \mathrm{~kg} / \mathrm{m}^{3}$.

Rheological properties of the outer coating:

$$
A=0,048 ; \quad \beta=0,05 ; \quad \alpha=0,1 .
$$

\section{Discussion}

Analysis of dispersion equations and their numerical solutions allows us to draw the following conclusions:

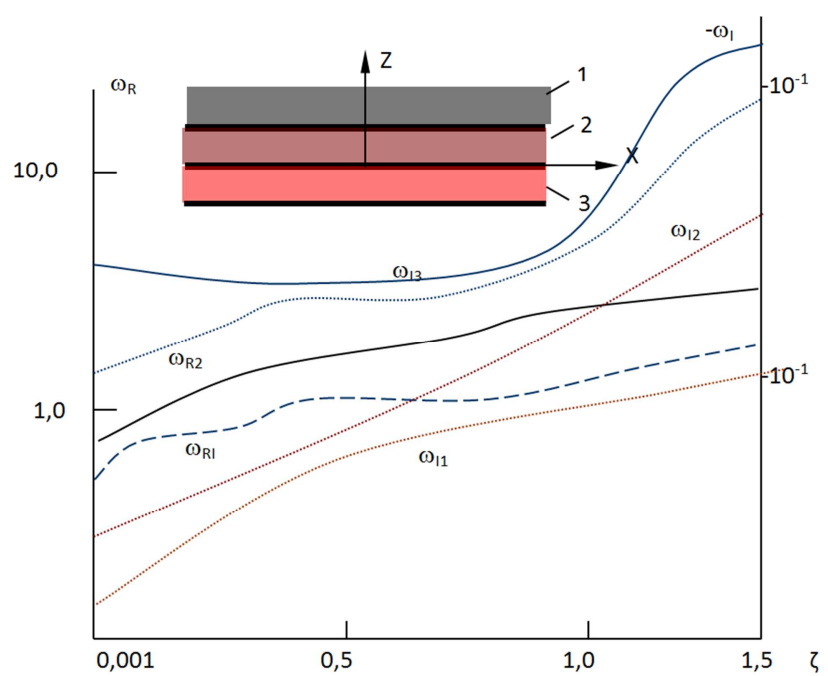

Figure 4. Change of natural frequencies from the wave number (dissipative homogeneous system).

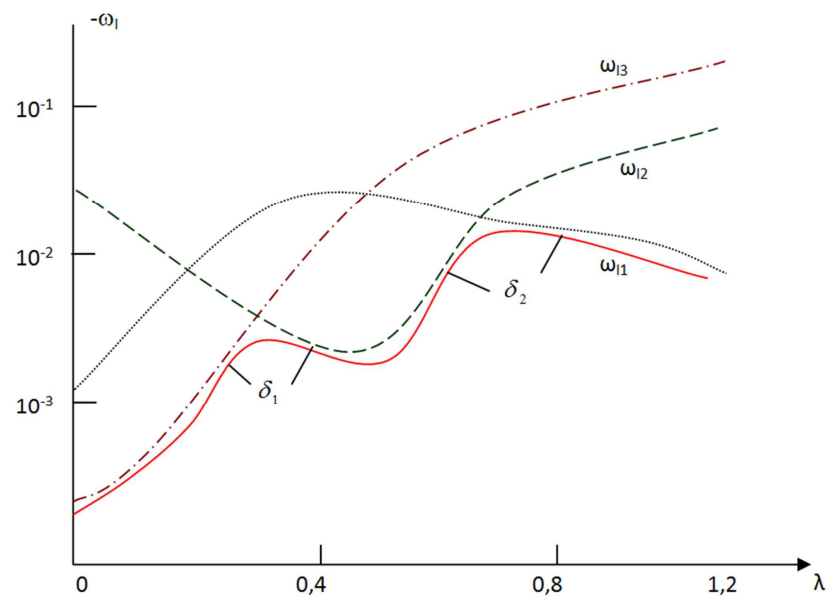

Figure 5. Change of natural frequencies from the wave number (dissipatively inhomogeneous system).

- for the dissipatively inhomogeneous mechanical systems, the "Troyanovskii-Safarov" effect [18] was found: the nonmonotonic dependence of the damping coefficients on the geometric and physico-mechanical parameters of mechanical systems;

- there is a symmetry of the dispersion curves when the complex wave number $\tilde{k}$ on $-\tilde{k}$;

- the larger the value of the parameter of the fractional exponential parameter of the nucleus $m$ and (or less the value $\beta$ ), the earlier the dispersion curves with the positive and negative imaginary parts begin to diverge $\tilde{k}$;

- with a decrease in the values of $\mathrm{m}$ and (or) with increasing values $\beta$ the behavior of the dispersion curves tends to the elastic case;

-dispersion curves of the hereditary-elastic spectrum corresponding to the real branches of the elastic spectrum are complex with a positive imaginary part $\tilde{k}$, which determines the attenuation of the coordinate solution;

- in the vicinity of the locking frequencies of the elastic spectrum, the branches of the hereditary-elastic spectrum have the greatest curvature. Increasing the values of $m$, like decreasing the values $\beta$, leads to a smoothing of the dispersion curves in these regions. Thus, the elastic spectrum can approximately be regarded as asymptotic for the hereditarily elastic $\mathrm{k} \rightarrow 0, \beta>1$.

\section{References}

[1] Kulesh M. A., Shardakov I. N. Wave dynamics of elastic media. PSU, Perm, 2007.- 60p.

[2] Kayumov S. S., Safarov I. I. Propagation and diffraction of waves in dissipative - inhomogeneous cylindrical deformable mechanical systems. Tashkent, 2002, 214p.

[3] Davies R. M. Stress waves in solids. Moscow, 1961, -104 p.

[4] Miker T., Meitzler A. Waveguide propagation in extended cylinders and plates. - Phys. Acoustics. Principles and methods. Trans. from English, 1966, 1 A, p. 140-203

[5] Natif A., Jones D., Henderson J. Dampening of vibrations: Persanal-M., 1968, 448 p.

[6] Safarov II, Teshaev M. Kh., Boltaev Z. I. Wave processes in a mechanical waveguide. LAP LAMBERT Academic publishing (Germany). 2012., 217 pp.

[7] Safarov I. I., Rashidov M., Kayumov S. S. Propagation of waves in dissipatively irregular planar bodies. International Conference "Aviation and Cosmonautics", 2006. Moscow p. 112-113.

[8] Seymov V. M., Trofimchuk O. A., Savitsky O. A. Vibrations and waves in layered media. -Kyiv: Science. Dumka, 1990.-224 p.

[9] Timoshenko S. P. Plates and shells. - Moscow: Nauka, 1966, $597 \mathrm{p}$.

[10] Achenbach J. D., Keshava S/P/ Free waves in a plate supted by a sani-infinite continuum -Trans. ASME. Ser.E J.Mech, 1967, V.34. \#2. p.398-404

[11] Safarov I. I., Boltaev Z. I., Akhmedov M. Distribution of the natural waves. LAP LAMBERT Academic Publishing Saarbrucren Dentschland /Germanu/-2015. -110p. 
[12] Safarov I. I., Akhmedov M., Rajabov O. Vibrations of plates and shells with attached concentrated mass. LAP LAMBERT Academic Publishing Saarbrucren Dentschland /Germanu/$2015-92 p$.

[13] Mirsaidov M. M., Troyanovsky I. E. Dynamics of inhomogeneous systems with allowance for internal dissipation and wave entrainment of energy. Tashkent: 1990. $170 \mathrm{p}$.

[14] Safarov I. I., Akhmedov M. S., Boltaev Z. I. DUCTING IN EXTENDED PLATES OF VARIABLE THICKNES. Sciences of Europe (Praha, Czech Republic). Vol 2, No 1 (1) (2016). p.21-39.

[15] Safarov I. I., Boltaev Z. I., Akhmedov M.Sh., Radzhabov O. I. Influence of the density of a liquid on the wave process in a viscoelastic fluid system. "The first independent scientific bulletin." Kiev, Monthly Scientific Journal, HF No. 2048910289RR, No. 6 / 2016. P. 109-121.

[16] Safarov I. I., Teshaev M. Kh., Boltaev Z. I., Axmedov M.Sh.Coommon natural in dissipative inhomogeous plane Bodies /Discovery, 2016,52,(251) 2108-2126.

[17] Hileo Saito, Kinchi Sato. Propagation of flexural waves and oscillations of multilayer rods and beams. Applied Mechanics. №2,1962.P.78-87.
[18] Teshaev M. Kh., Boltaev Z. I., Nuriddinov B. Z. Of Own and Forced Vibrations of Dissipative Inhomogeneous Mechanical Systems. Applied Mathematics, 2017, 8. P.1001-1015

[19] Rabotnov Yu. N. Elements of hereditary mechanics of solids. M.: Nauka press. 1977.383 p.

[20] Rzhanitsyn A. R. Creep theory. Moscow: Stroyizdat press, $1968.16 \mathrm{p}$.

[21] Koltunov M. A. To the problem of the choice of kernels in solving problems with allowance for creep and relaxation // Mechanics of polymers. 1966. №4. P. 483-497.

[22] Koltunov M. A., Mayboroda V. P., Zubchaninov V. G. Strength calculations of products made of polymer materials. M.: "Mashinastroyenie" press. 1983. 239 p.

[23] Korn G., Korn T. Handbook of Mathematics (for scientists and engineers). M.: Nauka press, 1984.- 832 p.

[24] Koltunov M. A. Creep and relaxation.-M: "Wisshaya shkola" press, 1976.- $276 \mathrm{p}$.

[25] Adamov A. A., Matveenko V. P., Trufanov N. A., Shardakov I. N. Methods of applied viscoelasticity. Ekaterinburg. 2003. $411 \mathrm{p}$. 